\title{
The transcriptional regulators RamA and RamB are involved in the regulation of glycogen synthesis in Corynebacterium glutamicum
}

Correspondence

Bernhard J. Eikmanns

bernhard.eikmanns@uni-ulm.de

Received 27 November 2009

Revised 23 December 2009

Accepted 30 December 2009

\author{
Gerd M. Seibold, ${ }^{1,2}$ Christian T. Hagmann, ${ }^{1}$ Melanie Schietzel, ${ }^{1}$ \\ Denise Emer, ${ }^{1}$ Marc Auchter, ${ }^{1}$ Joy Schreiner ${ }^{1}$ and Bernhard J. Eikmanns ${ }^{1}$ \\ ${ }^{1}$ Institute of Microbiology and Biotechnology, University of Ulm, D-89069 Ulm, Germany \\ ${ }^{2}$ Institute of Biochemistry, University of Cologne, D-50674 Cologne, Germany
}

\section{INTRODUCTION}

The Gram-positive bacterium Corynebacterium glutamicum is used for the industrial production of amino acids (Leuchtenberger et al., 2005). As in other bacteria, C. glutamicum synthesizes glycogen by the consecutive action of ADP-glucose pyrophosphorylase, glycogen synthase and glycogen branching enzyme, which are encoded by $g l g C$, $g l g A$ and $g l g B$, respectively (Tzvetkov et al., 2003; Seibold et al., 2007). In many bacteria, the formation of ADP-glucose from glucose-1-phosphate and ATP by ADP-glucose pyrophosphorylase is regarded as the key regulatory step of glycogen synthesis (Ballicora et al., 2003). In Escherichia coli, the regulation of this reaction takes place at several levels: transcription of $g l g C$ and $g l g A$ is activated by both the CRP/cAMP-dependent catabolite repression system and the stringent response (Romeo \& Preiss, 1989; Romeo et al., 1990). Translation and stability of the $g l g C-g l g A$ mRNA are regulated by the carbon storage regulation system (Baker et al., 2002; Weilbacher et al., 2003) and, at the post-translational level, bacterial ADP-glucose pyro-

Abbreviations: CAT, chloramphenicol acetyltransferase; dw, dry weight; EMSA, electrophoretic mobility shift assay; WT, wild-type. phosphorylases are generally allosterically controlled by various effector molecules (reviewed by Ballicora et al., 2003, 2007; Preiss et al., 1966).

We recently reported that during cultivation of $C$. glutamicum in medium containing carbohydrates (e.g. glucose, fructose or sucrose) the intracellular glycogen content reaches up to $10 \%$ of the cell's dry weight $(\mathrm{dw})$, whereas no glycogen is accumulated when the cells are cultivated with gluconeogenic substrates such as acetate (Seibold et al., 2007). Moreover, we showed that the first step of glycogen synthesis in C. glutamicum is regulated by controlling the activity of ADP-glucose pyrophosphorylase and by transcriptional regulation of $g l g C$ dependent on the carbon source (Seibold et al., 2007). However, the proteins involved in transcriptional control of $g l g C$ have not been identified so far.

The two transcriptional regulators RamA and RamB have been proposed to be master regulators for the adjustment of the central metabolism towards the utilization of gluconeogenic substrates for C. glutamicum (reviewed by Arndt \& Eikmanns, 2008). In the presence of acetate, RamA activates the expression of genes encoding proteins required for the utilization of acetate, such as the genes for 
isocitrate lyase, malate synthase, phosphotransacetylase and acetate kinase (aceA, aceB and pta-ack, respectively) (Cramer et al., 2006). Recently, RamA has also been shown to activate the adhA and ald genes, encoding alcohol and acetaldehyde dehydrogenases (Arndt \& Eikmanns, 2007; Auchter et al., 2009), the monocarboxylic acid transporter gene $m c t C$ (Jolkver et al., 2009), some further genes encoding enzymes of the central metabolism (Emer et al., 2009; Toyoda et al., 2009; Bussmann et al., 2009) and the $\operatorname{csp} B$ and $r p f 2$ genes, which encode the S-layer protein PS2 and a resuscitation factor, respectively (Hansmeier et al., 2006; Jungwirth et al., 2008). Furthermore, RamA has been shown to negatively regulate the expression of its own gene (Cramer \& Eikmanns, 2007) and to activate transcription of the ramB gene (Cramer et al., 2007). RamB is a negative regulator of the aceA, aceB, pta-ack, adhA, ald and $r p f 2$ genes (Gerstmeir et al., 2004; Arndt \& Eikmanns, 2007; Auchter et al., 2009; Jungwirth et al., 2008). Additionally, RamB is subject to negative autoregulation (Cramer et al., 2007) and acts as an activator of the pyruvate dehydrogenase complex subunit E1p gene aceE (Blombach et al., 2009).

Here, we present data showing the direct involvement of RamA in transcriptional activation of the ADP-glucose pyrophosphorylase and glycogen synthase genes $g \lg C$ and $g l g A$, and thus in glycogen synthesis of C. glutamicum. Furthermore, we show that RamB is also involved in expression control of $g \lg A$ and thus adjusts the glycogen synthesis rate in C. glutamicum.

\section{METHODS}

Bacterial strains, plasmids, oligonucleotides and growth conditions. Bacterial strains and plasmids used in this study are listed in
Table 1, and primers are listed in Table 2. E. coli and all C. glutamicum pre-cultures were grown aerobically in TY complex medium (Sambrook et al., 2001) at $37^{\circ} \mathrm{C}$ and $30^{\circ} \mathrm{C}$, respectively, as $60 \mathrm{ml}$ cultures in $500 \mathrm{ml}$ baffled Erlenmeyer flasks on a rotary shaker at 120 r.p.m. For the main C. glutamicum cultures, cells of an overnight pre-culture were washed twice with $0.9 \%(\mathrm{w} / \mathrm{v}) \mathrm{NaCl}$ and then inoculated into CGC minimal medium (Eikmanns et al., 1991) containing glucose and/or acetate at concentrations indicated in Results and Discussion. In minimal medium, C. glutamicum was grown aerobically at $30{ }^{\circ} \mathrm{C}$ as $50 \mathrm{ml}$ cultures in $500 \mathrm{ml}$ baffled Erlenmeyer flasks on a rotary shaker at 120 r.p.m. The growth of $E$. coli and C. glutamicum was followed by measuring the OD at $600 \mathrm{~nm}$.

DNA preparation, transformation and manipulation. Standard procedures were employed for plasmid isolation and for molecular cloning and transformation of E. coli $\mathrm{DH} 5 \alpha$, as well as for electrophoresis (Sambrook et al., 2001). C. glutamicum chromosomal DNA was isolated according to the method described by Eikmanns et al. (1994). Transformation of C. glutamicum was performed by electroporation using the methods described by Tauch et al. (2002); the recombinant strains were selected on Luria-Bertani-BHIS agar plates containing kanamycin $\left(25 \mu \mathrm{g} \mathrm{ml}^{-1}\right)$. E. coli was electroporated according to the method described by Dower et al. (1988). All restriction enzymes, T4-DNA ligase, shrimp alkaline phosphatase and Taq DNA polymerase were obtained from MBI Fermentas and used according to the manufacturer's instructions.

Cloning the $\boldsymbol{g} \mathbf{I g A}$ promoter. The promoter probe vector pET2 was used to construct a transcriptional fusion of the $g l g A$ promoter to the promoterless cat gene. The $g l g A$ promoter fragment was amplified from chromosomal DNA from C. glutamicum wild-type (WT) by PCR with the primers PR-glgAC-for and PRAC2rev. The 434 bp PCR product, covering the region from $308 \mathrm{bp}$ upstream to $125 \mathrm{bp}$ downstream of the annotated $g l g A$ translational start codon, was digested with SalI and BamHI and ligated into the multiple cloning site in front of the cat gene in pET2, resulting in plasmid pET-PA. Successful cloning was verified by sequencing.

RNA techniques. RNA from exponentially growing cultures of $C$. glutamicum was prepared using the RNeasy kit (Qiagen) as described

Table 1. Strains and plasmids used in this study

\begin{tabular}{|c|c|c|}
\hline Strain or plasmid & Relevant characteristics & References/Source \\
\hline \multicolumn{3}{|l|}{ E. coli strain } \\
\hline $\mathrm{DH} 5 \alpha$ & $\begin{array}{l}\mathrm{F}^{-} \phi 80 \text { lacZ } \triangle \mathrm{M} 15 \Delta(\text { lacZYA-argF }) \mathrm{U} 169 \text { endA1 recA1 hsdR17 }\left(\mathrm{r}_{\mathrm{k}}^{-}, \mathrm{m}_{\mathrm{k}}{ }^{+}\right) \\
\text {supE44 thi-1 gyrA96 relA1 phoA }\end{array}$ & Hanahan (1983) \\
\hline BL21 (DE3) & $\operatorname{omp} T, h s d S B\left(r_{B}^{-} m_{B}{ }^{-}\right), g a l, d c m(D E 3)$ & Studier \& Moffatt (1986) \\
\hline \multicolumn{3}{|l|}{ C. glutamicum strain } \\
\hline WT & Wild-type strain ATCC 13032 & $\begin{array}{l}\text { American Type Culture } \\
\text { Collection }\end{array}$ \\
\hline RG1 & C. glutamicum WT with truncated $\mathrm{ramB}$ gene, shortened by 775 bp & Gerstmeir et al. (2004) \\
\hline RG2 & C. glutamicum WT with truncated ramA gene, shortened by 364 bp & Cramer et al. (2006) \\
\hline \multicolumn{3}{|c|}{0} \\
\hline pET2 & Promoter probe vector carrying the promoterless cat gene, $\operatorname{Kan}^{\mathrm{R}}$ & Vasicova et al. (1998) \\
\hline pET2-PC & pET2 containing the $g \lg C$ promoter fragment & Seibold et al. (2007) \\
\hline pET2-PA & pET2 containing the $g \lg A$ promoter fragment & This work \\
\hline pDrive-RACE-PRglgA & pDrive derivative containing the PCR-amplified $g \lg A$ fragment from the RACE assay & This work \\
\hline
\end{tabular}


Table 2. Oligonucleotides used in this study

\begin{tabular}{|lll|}
\hline Oligonucleotide & \multicolumn{1}{c|}{ Sequence $\left(\mathbf{5}^{\prime}-\mathbf{3}^{\prime}\right)^{*}$} & Purpose \\
\hline PR-glgAC-for & CGGGATCCTGCACCCATGCAGTGAAC & pET2-PA, intAC \\
PRAC2rev & ACGCGTCGACTCCGGAGTTCACCA & pET2-PA, intAC \\
RACE-glgC-SP1 & CAGTACCGCGATCTTGAG & $5^{\prime}$-RACE, cDNA synthesis \\
RACE-glgC-SP2 & CTGTAAGTTCCGCCGAATGG & $5^{\prime}$-RACE, nested primer \\
RACE-glgA-SP1 & GTCTTAATCGCAGGGTTC & $5^{\prime}$-RACE, cDNA synthesis \\
RACE-glgA-SP2 & ACCCATGCAGTGAACATC & $5^{\prime}$-RACE, nested primer \\
Oligonucleotide dT & GACCACGCGTATCGATGTCGACTTTTTTTT & $5^{\prime}$-RACE, amplification of \\
anchor primer & TTTTTTTTTV & dA-tailed cDNA \\
ramBp3b_forw & ACGCGTCGACGATGTGGCCCCGACCACGCCG & ramBp3b \\
ramBp3c_forw & ACGCGTCGACCTCAGTGCCAAGAGTGGTTA & ramBp3b, ramBp3c \\
ramBp_rev & ACTGAGGTGTTGCAAACTTGTTGATTTTCGCT & ramB \\
\hline
\end{tabular}

${ }^{\star}$ Restriction sites are underlined; $\mathrm{V}$ represents an A, C or G.

previously (Seibold et al., 2007). To identify the transcriptional start site of $g l g C$ with the $5^{\prime} / 3^{\prime}$-RACE kit from Roche Diagnostics, $3 \mu \mathrm{g}$ total RNA was used as a template for the specific $g l g C$ cDNA synthesis using primer RACE-glgC-SP1. The subsequent PCRs were performed using the primer pair RACE-glgC-SP2/oligonucleotide dT anchor primer (the latter is included in the kit). To identify the glgA start site, primers RACE-glgA-SP1 and RACE-glgA-SP2 were used. The purified PCR products were ligated into plasmid pDrive (Qiagen), resulting in recombinant plasmids pDrive-RACE-PRglgC and pDrive-RACEPRglgA. These plasmids were sequenced and the transcriptional start sites were deduced from the sequences obtained.

Enzyme assays. To determine chloramphenicol acetyltransferase (CAT) activities in cell extracts, C. glutamicum cells were grown in minimal medium to the exponential growth phase, washed twice in $20 \mathrm{ml} 20 \mathrm{mM}$ Tris/ $\mathrm{HCl}$ buffer, $\mathrm{pH} \mathrm{7.8,} \mathrm{and} \mathrm{resuspended} \mathrm{in} 1 \mathrm{ml}$ of the same buffer containing $10 \mathrm{mM} \mathrm{MgCl}_{2}$ and $1 \mathrm{mM}$ EDTA. The cell suspension was added to $2 \mathrm{ml}$ screw cap vials together with $250 \mathrm{mg}$ glass beads (150-212 $\mu \mathrm{m}$; Sigma-Aldrich) and mechanically disrupted with a RiboLyser (setting 6.5; Hybaid) six times for $25 \mathrm{~s}$ at $4{ }^{\circ} \mathrm{C}$ with intermittent cooling on ice for $2 \mathrm{~min}$. After disruption, glass beads and cellular debris were removed by two consecutive centrifugation steps $\left(13000 \mathrm{~g}, 4{ }^{\circ} \mathrm{C}, 10 \mathrm{~min}\right.$ and $45000 \mathrm{~g}, 4{ }^{\circ} \mathrm{C}$, $60 \mathrm{~min}$ ) and the supernatant was used to determine the specific CAT activity (Gerstmeir et al., 2004). The bicinchoninic acid protein assay kit (Pierce) was used to determine protein concentrations according to the manufacturer's instructions, with BSA as the standard.

Protein purification and electrophoretic mobility shift assays (EMSAs). RamA and RamB were synthesized as hexahistidyl-tagged fusion proteins and purified by $\mathrm{Ni}^{2+}$ affinity chromatography as described previously (Gerstmeir et al., 2004; Cramer et al., 2006). Binding of purified RamA and RamB was tested by EMSAs using DNA fragments generated by PCR and purified using the Nucleospin Extract kit (Macherey-Nagel). The fragment intAC, carrying the $g l g A-$ $g l g C$ intergenic region, was amplified using primers PR-glgAC-for and PRAC2rev. The $211 \mathrm{bp}$ fragment $\mathrm{ramBp} 3 \mathrm{~b}$ generated with the primers ramBp3b_forw and ramBp_rev was used as a negative control for RamA binding and as a positive control for RamB binding (Cramer et al., 2007). The $135 \mathrm{bp}$ fragment $\mathrm{ramBp} 3 \mathrm{c}$ served as negative control for RamB binding and was generated by PCR using the primers ramBp3c_forw and ramBp_rev (Cramer et al., 2007). In the binding assays, 5-30 ng of the fragments was incubated with various amounts of RamA or RamB (0-1 $\mu$ g; corresponds to a molar excess of protein over DNA of 0-250-fold in the case of RamA and of 0-180-fold in the case of RamB) in $20 \mu \mathrm{l} 10 \mathrm{mM}$ Tris/ $\mathrm{HCl}$ reaction buffer, $\mathrm{pH}$ 7.6, containing $50 \mathrm{mM} \mathrm{NaCl}, 1 \mathrm{mM}$ DTT, $1 \mathrm{mM}$ EDTA, $10 \%$ (w/v) glycerol and $1 \mu \mathrm{g}$ Poly[d(I-C)] for $20 \mathrm{~min}$ at room temperature. The mixture was separated on a $2 \%(\mathrm{w} / \mathrm{v})$ agarose gel in $1 \times$ TAE buffer (40 mM Tris/HCl, pH 7.5, $20 \mathrm{mM}$ acetate, $1 \mathrm{mM}$ EDTA) at $70 \mathrm{~V}$ and $80 \mathrm{~mA}$ and stained with ethidium bromide.

Analysis of intracellular carbohydrates. For enzymic analysis of intracellular polysaccharides in C. glutamicum, $5 \mathrm{ml}$ samples of cultures were harvested, cell extracts were prepared and glycogen content was determined with amyloglucosidase (Roche Diagnostics) as described previously (Seibold et al., 2007).

Computational analysis. Comparative genome analysis was performed using the MBGD platform (Uchiyama, 2007) and sequence analysis was done using CoryneRegNet (Baumbach et al., 2006, 2009).

\section{RESULTS AND DISCUSSION}

\section{Influence of inactivating $\operatorname{ram} A$ or $\operatorname{ram} B$ on the glycogen content of C. glutamicum}

To study the possible effects of RamA and RamB on glycogen metabolism in C. glutamicum, the WT and the ramA and ramB deletion mutants C. glutamicum RG2 and C. glutamicum RG1, respectively, were cultured in minimal medium containing either glucose, glucose plus acetate, or acetate as the carbon source, and the glycogen content was measured at different time points. As expected from earlier experiments, when C. glutamicum WT was cultivated with glucose as the carbon source during early exponential phase, glycogen accumulated, and decreased in the course of cultivation (Fig. 1a; Seibold et al., 2007). Less glycogen was present in the course of cultivation of C. glutamicum WT with glucose plus acetate (Fig. 1b) and only minor amounts of glycogen were found when cultivated with acetate as the sole carbon source (Fig. 1c). As shown before, the RamA-deficient C. glutamicum RG2 did not grow on acetate as the sole carbon source (Cramer et al., 2006). 

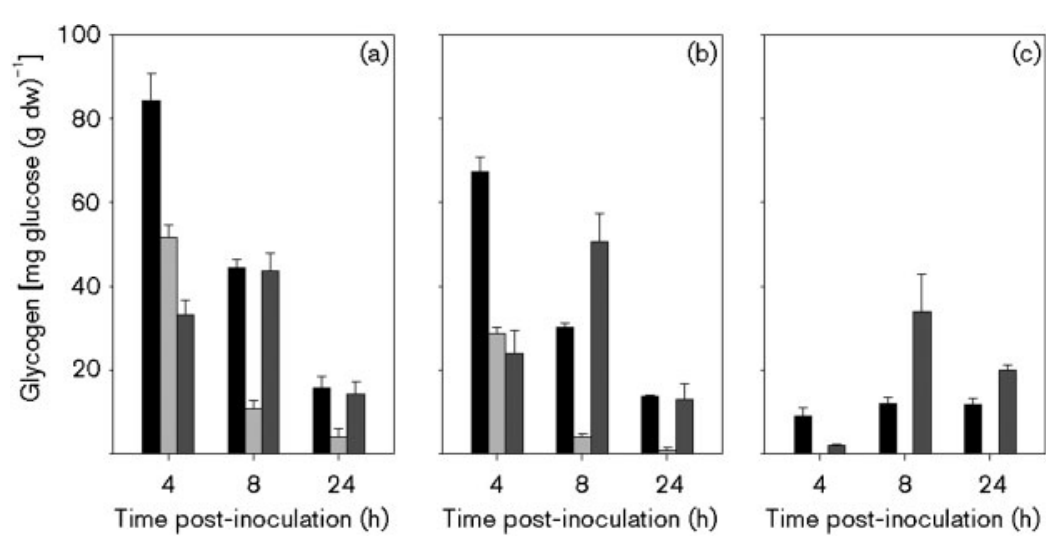

Fig. 1. Glycogen content (mean $\pm \mathrm{SD}$ ) of $C$. glutamicum WT (black bars), C. glutamicum RG2 (light grey) and C. glutamicum RG1 (dark grey) at selected time points during cultivation with $1 \%(\mathrm{w} / \mathrm{v})$ glucose (a), $0.5 \%$ $(\mathrm{w} / \mathrm{v})$ glucose plus $0.5 \%(\mathrm{w} / \mathrm{v})$ potassium acetate (b) or $1 \%(\mathrm{w} / \mathrm{v})$ potassium acetate (c). The pre-cultures were grown in TY complex medium, the glycogen content at $0 \mathrm{~h}$ was $<2 \mathrm{mg}(\mathrm{g} \mathrm{dw})^{-1}$ in all three strains. Three to five independent cultures were performed with two determinations per experiment.
However, when cultivated with glucose or with glucose plus acetate as carbon sources, the glycogen content in the RamA-deficient C. glutamicum RG2 was significantly lower than in C. glutamicum WT (Fig. 1a and b). These results indicate that RamA positively influences glycogen synthesis, possibly by transcriptional activation of $g \lg C$, which has been shown to be expressed in a carbon-sourcedependent manner (Seibold et al., 2007). However, although the glycogen content of C. glutamicum RG2 was lower than in the WT strain, it differed during cultivation on glucose with or without acetate (Fig. 1). This result indicates that RamA is not responsible for the carbonsource-dependent differences in glycogen content. The pattern observed for glycogen accumulation in the RamBdeficient mutant C. glutamicum RG1 is different from that of C. glutamicum WT, i.e. most glycogen was found in the late exponential growth phase ( $8 \mathrm{~h}$ after inoculation), independent of the carbon source used for cultivation (Fig. 1). Furthermore, the $\operatorname{ramB}$ mutant strain also accumulated significant amounts of glycogen when cultivated with acetate as the sole carbon source (Fig. 1c). These results indicate that in the presence of acetate in the growth medium, RamB might act as a repressor of genes encoding enzymes required for glycogen synthesis and is possibly responsible for the carbon-source-dependent regulation of glycogen content in C. glutamicum.

\section{Identifying the transcriptional start sites in the $g / g A-g / g C$ intergenic region and the putative binding sites for RamA and RamB}

The genes encoding the enzymes that catalyse the first two steps of glycogen synthesis, $g \lg C$ and $g \lg A$, are adjacent on the genome sequence of $C$. glutamicum, separated by $153 \mathrm{bp}$ and are divergently transcribed (Kalinowski et al., 2003; Seibold et al., 2007). Both genes are transcribed monocistronically (Seibold et al., 2007; data not shown). To determine the transcriptional start sites of both genes, we performed 5'-RACE with total RNA of both glucoseand acetate-grown C. glutamicum WT. After cDNA synthesis of the $5^{\prime}$-end transcripts of $g \lg A$ and $g \lg C$ with specific primers for each gene, the amplified cDNAs were subcloned in pDrive and the resulting plasmids were sequenced. In three independent experiments with RNA from cells cultivated with glucose as well as with acetate, the transcriptional start site of $\operatorname{glgC}$ (TSc) was found to be identical to the translational start site (see Fig. 2). Upstream of TSc, we found the motif TATGGT, which is identical in four of six bases to the -10 consensus motif (TAC/TAAT) described for corynebacteria (Pátek et al., 2003) and which is identical to the -10 motif described for the ald gene (Auchter et al., 2009). The transcriptional start site of $g l g A$ (TSa) was identified by three independent 5 '-RACE experiments and found to be the A residue of the annotated ATG start codon (see Fig. 2). A possible -10 motif (GCTAAT) would be the GCTAAT sequence, which is also conserved in four of six bases compared to the -10 consensus motif (Pátek et al., 2003). Overlap of transcriptional and translational start points was observed quite frequently in C. glutamicum (brnF, lrp, lpdA, ilvA, leuA, betP and several other genes) (Pátek et al., 2003); however, the interaction of the respective mRNAs with the 16S rRNA has not been investigated in $C$. glutamicum.

The known binding motifs for both transcriptional regulators RamA and RamB have recently been listed (reviewed by Arndt \& Eikmanns, 2008) and the derived consensus sequences were used to screen the $g \lg A-g \lg C$ intergenic region for such motifs. As shown in Fig. 2, three putative RamA binding sites (TGGGGGC, AGGGGC and ACCCCA) are located in the intergenic region, centred 52, 65 and 81 bp upstream of the $g \lg A$ transcriptional start site and 102, 89 and 73 bp upstream of the $g \operatorname{lgC}$ transcriptional start site, respectively. A putative RamB binding site (TAATCTTTGAAAT), consistent in 9 of 13 bases with the AA/GAACTTTGCAAA consensus RamB-binding motif (Gerstmeir et al., 2004), is located next to the $g \lg A$ transcriptional start site and overlaps the putative -10 region of $\operatorname{glg} A$ (see Fig. 2). Further analysis of the $g \lg A-g \lg C$ intergenic region for putative binding sites of other known corynebacterial transcriptional regulators (using the CoryneRegNet database; Baumbach et al., 2006, 2009) revealed no binding site of high significance apart from those described for RamA and RamB. 


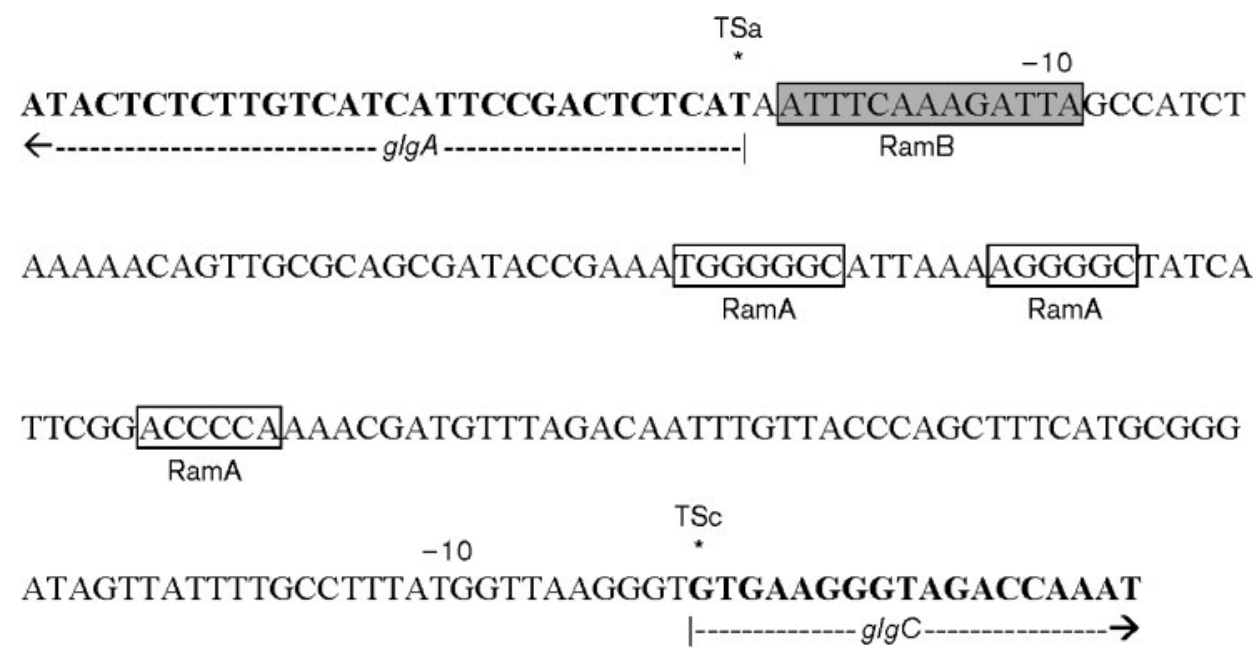

Fig. 2. Genomic locus of the intergenic region of the C. glutamicum glgA and glgC genes. Coding regions are shown in bold. Putative RamA binding sites are boxed, the putative RamB binding site is highlighted by a grey box, and the $g l g A$ (TSa) and glgC (TSc) transcriptional start sites are indicated by asterisks. The -10 regions are indicated above the sequence.

\section{RamA is a positive regulator of $g / g A$ and $g / g C$ transcription}

EMSAs were performed to confirm the binding of RamA to the $g \lg A-g \lg C$ intergenic region postulated above. For this purpose, different amounts of purified hexahistidyl-tagged RamA fusion protein $\left(\mathrm{RamA}^{\mathrm{HIS}}\right)$ were incubated with the intAC DNA fragment, which comprises the intergenic $g \lg C-g \lg A$ region. As shown in Fig. 3, the intAC probe was already slightly retarded by incubation with $0.15 \mu \mathrm{g}$ RamA; an increase in $\operatorname{Ram}^{\mathrm{HIS}}$ concentration to $0.65 \mu \mathrm{g}$ caused a complete shift. It is evident that two DNA-RamA complexes were formed, possibly reflecting the binding to two binding sites on the DNA fragment used. No retardation was observed with the control fragment ramBp3b, which possesses no RamA binding site (Cramer et al., 2007) and which was added as a negative control to the reaction mixtures (Fig. 3). From these results, we conclude that RamA specifically interacts with the $g \lg A-g \lg C$ intergenic region.

To test for transcriptional regulation of $\operatorname{glg} C$ and $g \lg A$ by RamA in vivo, transcriptional fusions between the pro- moter regions of both genes and the promoterless CAT gene in the promoter probe vector pET2 (i.e. plasmids pET-PC and pET-PA) were transformed into C. glutamicum WT and the ramA deletion mutant C. glutamicum RG2. CAT activities were determined in the plasmidcarrying strains during early exponential growth in minimal medium with $1 \%(\mathrm{w} / \mathrm{v})$ glucose, $0.5 \%(\mathrm{w} / \mathrm{v})$ glucose plus $0.5 \%(\mathrm{w} / \mathrm{v})$ potassium acetate or $1 \%(\mathrm{w} / \mathrm{v})$ potassium acetate. As already indicated by previous CAT reporter studies with $g \lg C$ (Seibold et al., 2007), the $g \operatorname{lgC}$ promoter activity varied in a carbon-source-dependent manner in C. glutamicum WT. The activity was highest in cells cultivated with glucose and was lower when acetate was the only, or an additional, carbon source (Table 3). The $g \lg A$ promoter activities varied in a similar way in $C$. glutamicum WT cells (Table 3). Independent of the carbon source (glucose or acetate, respectively), the promoter activities for both $g \lg C$ and $g \lg A$ were 30-70\% lower in the ramA-deficient mutant $C$. glutamicum RG2. Due to the direct interaction of RamA ${ }^{\text {HIS }}$ with the intAC fragment, the reduced glycogen content and the reduced $g \lg C$ and $g \lg A$ promoter activities in the $\operatorname{ram} A$ deletion strain $C$.

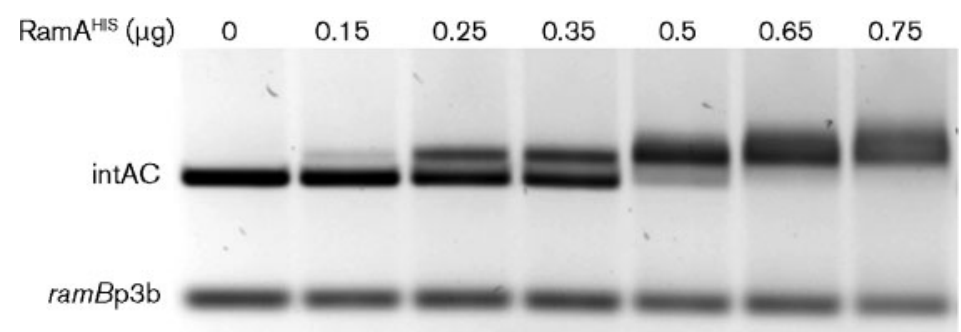

Fig. 3. Representative EMSA using hexahistidyl-tagged RamA protein $(0,0.15,0.25,0.35$, $0.5,0.65$ and $0.75 \mu \mathrm{g})$, with the $0.46 \mathrm{~kb}$ int $A C$ fragment as a probe (10 $\mathrm{ng}$ ) and the $0.21 \mathrm{~kb}$ ramBp3b fragment $(5 \mathrm{ng})$ as a negative control. 
Table 3. Specific CAT activities of C. glutamicum WT, RG2 and RG1 cells carrying the glgC or glgA promoter fragment in plasmid pET2 and cultivated in minimal medium containing $1 \%(\mathrm{w} / \mathrm{v})$ glucose, $1 \%(\mathrm{w} / \mathrm{v})$ potassium acetate, or $0.5 \%(\mathrm{w} / \mathrm{v}) \mathrm{glucose}$ plus $0.5 \%$ $(\mathrm{w} / \mathrm{v})$ potassium acetate as carbon source

All values are mean $\pm \mathrm{SD}$ of three to five independent experiments and two determinations per experiment.

\begin{tabular}{|c|c|c|c|}
\hline \multirow[t]{2}{*}{ Strain } & \multicolumn{3}{|c|}{ Specific CAT activity $\left[\mathrm{U}(\mathrm{mg} \text { protein })^{-1}\right]$} \\
\hline & Glucose & Glucose + acetate & Acetate \\
\hline C. glutamicum RG2 (pET2-PC) & $0.10 \pm 0.01$ & $0.05 \pm 0.00$ & No growth* \\
\hline C. glutamicum RG1 (pET2-PC) & $0.14 \pm 0.01$ & $0.11 \pm 0.02$ & $0.09 \pm 0.01$ \\
\hline C. glutamicum WT (pET2-PA) & $0.10 \pm 0.01$ & $0.09 \pm 0.01$ & $0.07 \pm 0.00$ \\
\hline
\end{tabular}

${ }^{\star}$ The RamA-deficient C. glutamicum RG2 and plasmid-carrying derivatives did not grow on acetate as the sole carbon source (Cramer et al., 2006).

glutamicum RG2, we conclude that RamA is a transcriptional activator of both $g \lg A$ and $g \lg C$ and thus is a positive regulator of glycogen synthesis in C. glutamicum.

\section{RamB is a negative transcriptional regulator of $g / g A$}

The interaction of $\mathrm{RamB}$ with the $g \lg A-g \lg C$ intergenic region was also investigated with EMSAs. As shown in Fig. 4 , a slight retardation of the intAC probe was observed when $0.25 \mu \mathrm{g}$ purified hexahistidyl-tagged RamB fusion protein $\left(\mathrm{RamB}^{\mathrm{HIS}}\right)$ was used in the assay. However, an increase in $\mathrm{RamB}^{\mathrm{HIS}}$ to up to $1 \mu \mathrm{g}$ in the EMSA did not cause a complete shift of the probe, indicating a rather weak binding of $\mathrm{RamB}^{\mathrm{HIS}}$ to the DNA fragment. From these data, we conclude that, in spite of four mismatches in the 13 bp RamB consensus sequence, the postulated RamB binding motif between the -10 region and the transcriptional start site is sufficient to bind RamB.

To analyse the effects of RamB on the $g \lg A$ and $g \operatorname{lgC}$ promoter activities in vivo, the promoter probe vectors pET-PC and pET-PA were transformed into the RamBdeficient mutant C. glutamicum RG1 and CAT activities were determined. The $g \lg C$ and $g \lg A$ promoter activities in the $\operatorname{ram} B$ deletion mutant varied depending on the carbon source in the same way as in C. glutamicum WT cells (Table 3). As no differences in transcription activity were observed for glgC in C. glutamicum WT and C. glutamicum RG1 cells (Table 3), we conclude that RamB is not involved in the transcriptional regulation of $g \operatorname{lgC}$. However, on all media tested, the transcription activity of $\operatorname{glg} A$ was about $50 \%$ higher in the RamB-deficient mutant C. glutamicum RG1 than in C. glutamicum WT. These data, in combination with the localization of the RamB binding site near the $g \lg A$ translational/transcriptional start site (see Fig. 2) and the results of the EMSA experiments (Fig. 4), indicate that RamB acts as a repressor of $g \lg A$ transcription.

\section{Conclusion}

Taken together, our data show that RamA and RamB are involved in the transcriptional regulation of $\operatorname{glg} C$ and $\operatorname{glg} A$ and thus in control of glycogen synthesis in C. glutamicum. Whereas RamA positively controls both $g \lg C$ and $g \lg A$, RamB negatively controls only the latter. The gene arrangement of $g \lg C$ and $g \lg A$ as adjoining but divergently transcribed genes in C. glutamicum allows differential expression of both genes and is similar to the gene arrangements present in other glycogen-synthesizing Corynebacterineae, such as Mycobacterium tuberculosis, Mycobacterium smegmatis, Mycobacterium phlei and Rhodococcus jostii (Sambou et al., 2008; Elbein \& Mitchell, 1973; Antoine \& Tepper, 1969; Hernández et al., 2008). In contrast, the two genes are organized as operonlike structures in other glycogen-synthesizing bacteria, e.g.

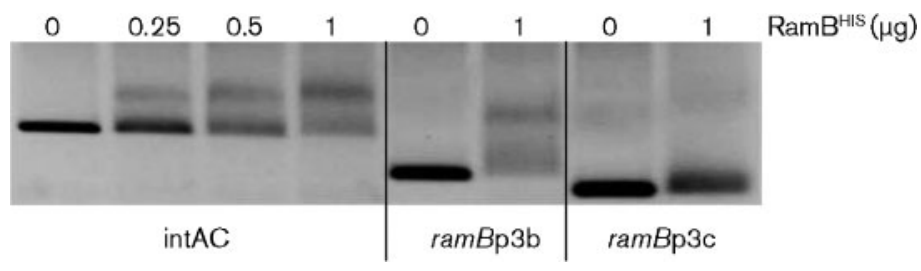

Fig. 4. Representative EMSA using hexahistidyl-tagged RamB protein $(0,0.25,0.5$ and $1 \mu \mathrm{g})$, with the $0.46 \mathrm{~kb}$ intAC fragment $(10 \mathrm{ng})$ as a probe and fragments ramBp3b and $\mathrm{ramBp} 3 \mathrm{c}$ (10 $\mathrm{ng}$ each) as positive and negative controls, respectively. The fragments used for the binding assays are indicated below the different sections of the gel. 
E. coli, Bacillus subtilis, Agrobacterium tumefaciens, Vibrio cholerae and Rhodobacter sphaeroides (Romeo et al., 1988; Kiel et al., 1994; Ugalde et al., 1998; Bourassa \& Camilli, 2009; Igarashi \& Meyer, 2000), and thus, they are co-ordinately controlled at the transcriptional level in these bacteria. In view of the finding that glycogen synthesis in most bacteria, and probably also in C. glutamicum, is regulated mainly by controlling the activity of ADP-glucose pyrophosphorylase (Ballicora et al., 2003; Seibold et al., 2007), the relatively weak differential expression control of $g \lg A$ and $g l g C$ by RamA and RamB possibly serves as a finetuning of glycogen metabolism. However, in contrast with RamA- and RamB-mediated transcription control of aceA, aceB, pta-ack, adhA, acn and gapA (Arndt \& Eikmanns, 2008; Emer et al., 2009; Toyoda et al., 2009), both RamAmediated activation of $g \lg C$ and $g \lg A$ and RamB-mediated repression of $g l g A$ are not dependent on the carbon sources tested. Thus, the situation is similar to that of RamA-/ RamB-mediated expression control of the $s d h C A B$ operon and the ald gene (Auchter et al., 2009; Bussmann et al., 2009); it becomes clear that a further transcriptional regulator(s) should be involved in carbon-source-dependent regulation of $g \operatorname{lgC}$ and $g \operatorname{lgA}$. However, in spite of a DNA affinity approach (data not shown) we were so far not able to identify a further regulator involved in $g \lg C$ and $g \lg A$ expression control.

\section{ACKNOWLEDGEMENTS}

The authors thank Ute Meyer and Eva Glees for excellent technical assistance. The support of the BMBF (grants 0313704 'SysMAP' and 0313805G 'GenoMik-Plus') and of Evonik Degussa GmbH, HalleKünsebeck, is gratefully acknowledged.

\section{REFERENCES}

Antoine, A. D. \& Tepper, B. S. (1969). Environmental control of glycogen and lipid content of Mycobacterium phlei. Microbiology 55, 217-226.

Arndt, A. \& Eikmanns, B. J. (2007). The alcohol dehydrogenase gene adhA in Corynebacterium glutamicum is subject to carbon catabolite repression. J Bacteriol 189, 7408-7416.

Arndt, A. \& Eikmanns, B. J. (2008). Regulation of carbon metabolism in Corynebacterium glutamicum. In Corynebacteria: Genomics and Molecular Biology, pp. 155-182. Edited by A. Burkovski. Norfolk, UK: Caister Academic Press.

Auchter, M., Arndt, A. \& Eikmanns, B. J. (2009). Dual transcriptional control of the acetaldehyde dehydrogenase gene ald of Corynebacterium glutamicum by RamA and RamB. J Biotechnol 140, 84-91.

Baker, C. S., Morozov, I., Suzuki, K., Romeo, T. \& Babitzke, P. (2002). CsrA regulates glycogen biosynthesis by preventing translation of $g l g C$ in Escherichia coli. Mol Microbiol 44, 1599-1610.

Ballicora, M. A., Iglesias, A. A. \& Preiss, J. (2003). ADP-Glucose pyrophosphorylase, a regulatory enzyme for bacterial glycogen synthesis. Microbiol Mol Biol Rev 67, 213-225.

Ballicora, M. A., Erben, E. D., Yazaki, T., Bertolo, A. L., Demonte, A. M., Schmidt, J. R., Aleanzi, M., Bejar, C. M., Figueroa, C. M. \& other authors (2007). Identification of regions critically affecting kinetics and allosteric regulation of the Escherichia coli ADP-glucose pyrophosphorylase by modelling and pentapeptide-scanning mutagenesis. J Bacteriol 189, 5325-5333.

Baumbach, J., Brinkrolf, K., Czaja, L. F., Rahmann, S. \& Tauch, A. (2006). CoryneRegNet: an ontology-based data warehouse of corynebacterial transcription factors and regulatory networks. $B M C$ Genomics 7, 24.

Baumbach, J., Wittkop, T., Kleindt, C. K. \& Tauch, A. (2009). Integrated analysis and reconstruction of microbial transcriptional gene regulatory networks using CoryneRegNet. Nat Protoc 4, 9921005.

Blombach, B., Cramer, A., Eikmanns, B. J. \& Schreiner, M. (2009). RamB is an activator of the pyruvate dehydrogenase complex subunit E1p gene in Corynebacterium glutamicum. J Mol Microbiol Biotechnol 16, 236-239.

Bourassa, L. \& Camilli, A. (2009). Glycogen contributes to the environmental persistence and transmission of Vibrio cholerae. Mol Microbiol 72, 124-138.

Bussmann, M., Emer, D., Hasenbein, S., Degraf, S., Eikmanns, B. J. \& Bott, M. (2009). Transcriptional regulation of the succinate dehydrogenase operon $s d h C A B$ of Corynebacterium glutamicum by the cAMP-dependent regulator GlxR and the LuxR-type regulator RamA. J Biotechnol 143, 173-182.

Cramer, A. \& Eikmanns, B. J. (2007). RamA, the transcriptional regulator of acetate metabolism in Corynebacterium glutamicum, is subject to negative autoregulation. J Mol Microbiol Biotechnol 12, 5159.

Cramer, A., Gerstmeir, R., Schaffer, S., Bott, M. \& Eikmanns, B. J. (2006). Identification of RamA, a novel LuxR-type transcriptional regulator of genes involved in acetate metabolism of Corynebacterium glutamicum. J Bacteriol 188, 2554-2567.

Cramer, A., Auchter, A., Frunzke, J., Bott, M. \& Eikmmans, B. J. (2007). RamB, the transcriptional regulator of acetate metabolism in Corynebacterium glutamicum, is subject to regulation by RamA and RamB. J Bacteriol 189, 1145-1149.

Dower, W. J., Miller, J. F. \& Ragsdale, C. W. (1988). High efficiency transformation of Escherichia coli by high voltage electroporation. Nucleic Acids Res 16, 6127-6145.

Eikmanns, B. J., Metzger, M., Reinscheid, D., Kircher, M. \& Sahm, H. (1991). Amplification of three threonine biosynthesis genes in Corynebacterium glutamicum and its influence on carbon flux in different strains. Appl Microbiol Biotechnol 34, 617-622.

Eikmanns, B. J., Thum-Schmitz, N., Eggeling, L., Ludtke, K. U. \& Sahm, H. (1994). Nucleotide sequence, expression and transcriptional analysis of the Corynebacterium glutamicum gltA gene encoding citrate synthase. Microbiology 140, 1817-1828.

Elbein, A. D. \& Mitchell, M. (1973). Levels of glycogen and trehalose in Mycobacterium smegmatis and the purification and properties of the glycogen synthetase. J Bacteriol 113, 863-873.

Emer, D., Krug, A., Eikmanns, B. J. \& Bott, M. (2009). Complex expression control of the Corynebacterium glutamicum aconitase gene: identification of RamA as a third transcriptional regulator besides AcnR and RipA. J Biotechnol 140, 92-98.

Gerstmeir, R., Cramer, A., Dangel, P., Schaffer, S. \& Eikmanns, B. J. (2004). RamB, a novel transcriptional regulator of genes involved in acetate metabolism of Corynebacterium glutamicum. J Bacteriol 186, 2798-2809.

Hanahan, D. (1983). Studies on transformation of Escherichia coli with plasmids. J Mol Biol 166, 557-580.

Hansmeier, N., Albersmeier, A., Tauch, A., Damberg, T., Ros, R., Anselmetti, D., Pühler, A. \& Kalinowski, J. (2006). The surface (S)layer gene $\operatorname{csp} B$ of Corynebacterium glutamicum is transcriptionally 
activated by a LuxR-type regulator and located on a $6 \mathrm{~kb}$ genomic island absent from the type strain ATCC 13032. Microbiology 152, 923-935.

Hernández, M. A., Mohn, W. W., Martínez, E., Rost, E., Alvarez, A. F. \& Alvarez, H. M. (2008). Biosynthesis of storage compounds by Rhodococcus jostii RHAl and global identification of genes involved in their metabolism. BMC Genomics 9, 600.

Igarashi, R. Y. \& Meyer, C. R. (2000). Cloning and sequencing of glycogen metabolism genes from Rhodobacter sphaeroides 2.4.1. Expression and characterization of recombinant ADP-glucose pyrophosphorylase. Arch Biochem Biophys 376, 47-58.

Jolkver, E., Emer, D., Ballan, S., Krämer, R., Eikmanns, B. J. \& Marin, K. (2009). Identification and characterization of a bacterial transport system for the uptake of pyruvate, propionate, and acetate in Corynebacterium glutamicum. J Bacteriol 191, 940-948.

Jungwirth, B., Emer, D., Brune, I., Hansmeier, N., Pühler, A., Eikmanns, B. J. \& Tauch, A. (2008). Triple transcriptional control of the resuscitation promoting factor 2 (rpf2) gene of Corynebacterium glutamicum by the regulators of acetate metabolism RamA and RamB and the cAMP-dependent regulator GlxR. FEMS Microbiol Lett 281, 190-197.

Kalinowski, J., Bathe, B., Bartels, D., Bischoff, N., Bott, M., Burkovski, A., Dusch, N., Eggeling, L., Eikmanns, B. J. \& other authors (2003). The complete Corynebacterium glutamicum ATCC 13032 genome sequence and its impact on the production of L-aspartate-derived amino acids and vitamins. J Biotechnol 104, 5-25.

Kiel, J. A., Boels, J. M., Beldman, G. \& Venema, G. (1994). Glycogen in Bacillus subtilis: molecular characterisation of an operon encoding enzymes involved in glycogen biosynthesis and degradation. Mol Microbiol 11, 203-218.

Leuchtenberger, W., Huthmacher, K. \& Drauz, K. (2005). Biotechnological production of amino acids and derivatives: current status and prospects. Appl Microbiol Biotechnol 69, 1-8.

Pátek, M., Nešvera, J., Guyonvarch, A., Reyes, O. \& Leblon, G. (2003). Promoters of Corynebacterium glutamicum. J Biotechnol 104, 311-323.

Preiss, J., Shen, E., Greenberg, E. \& Gentner, N. (1966). Biosynthesis of bacterial glycogen. IV. Activation and inhibition of the adenosine diphosphate glucose pyrophosphorylase of Escherichia coli B. Biochemistry 5, 1833-1845.

Romeo, T. \& Preiss, J. (1989). Genetic regulation of glycogen biosynthesis in Escherichia coli: In vitro effects of cyclic AMP and guanosine $5^{\prime}$-diphosphate 3 'diphosphate and analysis of in vivo transcripts. J Bacteriol 171, 2773-2782.

Romeo, T., Kumar, A. \& Preiss, J. (1988). Analysis of the Escherichia coli glycogen gene cluster suggests that catabolic enzymes are encoded among the biosynthetic genes. Gene 70, 363-376.

Romeo, T., Black, J. \& Preiss, J. (1990). Genetic regulation of glycogen biosynthesis in Escherichia coli: in vivo effects of the catabolite repression and stringent response systems in glg gene expression. Curr Microbiol 21, 131-137.

Sambou, T., Dinadayala, P., Stadthagen, G., Barilone, N., Bordat, Y., Constant, P., Levillain, F., Neyrolles, O., Gicquel, B. \& other authors (2008). Capsular glucan and intracellular glycogen of Mycobacterium tuberculosis: biosynthesis and impact on the persistence in mice. Mol Microbiol 70, 762-774.

Sambrook, J., Russel, D. W., Irwin, N. \& Janssen, U. A. (2001). Molecular Cloning: a Laboratory Manual, 3rd edn. Cold Spring Harbor, NY: Cold Spring Harbor Laboratory.

Seibold, G., Dempf, S., Schreiner, J. \& Eikmanns, B. J. (2007). Glycogen formation in Corynebacterium glutamicum and role of ADPglucose pyrophosphorylase. Microbiology 153, 1275-1285.

Studier, F. W. \& Moffatt, B. A. (1986). Use of bacteriophage T7 RNA polymerase to direct selective high-level expression of cloned genes. J Mol Biol 189, 113-130.

Tauch, A., Homann, l., Mormann, S., Rüberg, S., Billault, A., Bathe, B., Brand, S., Brockmann-Gretza, O., Rückert, C. \& other authors (2002). Strategy to sequence the genome of Corynebacterium glutamicum ATCC 13032: use of a cosmid and a bacterial artificial chromosome library. J Biotechnol 95, 25-38.

Toyoda, K., Teramoto, H., Inui, M. \& Yukawa, H. (2009). Involvement of the LuxR-type transcriptional regulator RamA in regulation of expression of the gap gene, encoding glycerinaldehyde-3-phosphate dehydrogenase in Corynebacterium glutamicum. J Bacteriol 191, 968977.

Tzvetkov, M., Klopprogge, C., Zelder, O. \& Liebl, W. (2003). Genetic dissection of trehalose biosynthesis in Corynebacterium glutamicum: inactivation of trehalose production leads to impaired growth and an altered cell wall composition. Microbiology 149, 1659-1673.

Uchiyama, I. (2007). MBGD: a platform for microbial genomics based on the automated construction of orthologous groups. Nucleic Acids Res 35, D343-D346.

Ugalde, J. E., Lepek, V., Uttaro, A., Estrella, J., Iglesias, A. \& Ugalde, R. A. (1998). Gene organization and transcript analysis of the Agrobacterium tumefaciens glycogen $(\mathrm{glg})$ operon: two transcripts for the single phosphoglucomutase gene. J Bacteriol 180, 65576564.

Vasicova, P., Abrhamova, Z., Nesvera, J., Patek, M., Sahm, H. \& Eikmanns, B. J. (1998). Integrative and autonomously replicating vectors for analysis of promoters in Corynebacterium glutamicum. Biotechnol Tech 12, 743-746.

Weilbacher, T., Suzuki, K., Dubey, A. K., Wang, X., Gudapaty, S., Morozov, I., Baker, C. S., Georgellis, D., Babitzke, P. \& Romeo, T. (2003). A novel sRNA component of the carbon storage regulatory system of Escherichia coli. Mol Microbiol 48, 657-670.

Edited by: L. Heide 